\title{
Single-spin qubit magnetic spectroscopy of two-dimensional superconductivity
}

\author{
Shubhayu Chatterjee $\odot,{ }^{1, *}$ Pavel E. Dolgirev $\odot,{ }^{2, *}$ Ilya Esterlis $\odot,{ }^{2}$ Alexander A. Zibrov, ${ }^{2}$ Mikhail D. Lukin, ${ }^{2}$ \\ Norman Y. Yao, ${ }^{1,3}$ and Eugene Demler ${ }^{2,4}$ \\ ${ }^{1}$ Department of Physics, University of California, Berkeley, California 94720, USA \\ ${ }^{2}$ Department of Physics, Harvard University, Cambridge, Massachusetts 02138, USA \\ ${ }^{3}$ Materials Sciences Division, Lawrence Berkeley National Laboratory, Berkeley, California 94720, USA \\ ${ }^{4}$ Institute for Theoretical Physics, ETH Zurich, 8093 Zurich, Switzerland
}

(Received 28 June 2021; revised 3 December 2021; accepted 17 December 2021; published 10 January 2022)

\begin{abstract}
A single-spin qubit placed near the surface of a conductor acquires an additional contribution to its $1 / T_{1}$ relaxation rate due to magnetic noise created by electric current fluctuations in the material. We analyze this technique as a wireless probe of superconductivity in atomically thin two-dimensional materials. At temperatures $T \lesssim T_{c}$, the dominant contribution to the qubit relaxation rate is due to transverse electric current fluctuations arising from quasiparticle excitations. We demonstrate that this method enables detection of metal-to-superconductor transitions, as well as investigation of the symmetry of the superconducting gap function, through the noise scaling with temperature. We show that scaling of the noise with sample-probe distance provides a window into the nonlocal quasistatic conductivity of superconductors, both clean and disordered. At low temperatures the quasiparticle fluctuations get suppressed, yet the noise can be substantial due to resonant contributions from collective longitudinal modes, such as plasmons in monolayers and Josephson plasmons in bilayers. Potential experimental implications are discussed.
\end{abstract}

DOI: 10.1103/PhysRevResearch.4.L012001

Introduction. A superconductor is a phase of matter characterized by the dissipation-free flow of electrical current owing to the intrinsic quantum coherence between electron pairs [1]. The recent discovery of robust superconductivity in a variety of two-dimensional (2D) materials, such as moiré graphene [2-6], transition metal dichalcogenides (TMDs) [7], and monolayer FeSe [8-10], has spawned immense theoretical and experimental interest. The experimental tunability of 2D materials, e.g., in situ varying of carrier density via gate voltages, bandwidth using displacement fields, and dielectric properties/screening with substrates, makes them highly relevant for practical applications. However, while superconductivity in bulk solids and thin films has been extensively studied [1], characterizing it in bona fide 2D materials poses a significant challenge. In certain materials like TMDs, superconductivity itself can be hard to detect due to the difficulty of making contacts for transport experiments [11]. Conventional bulk probes of the nature of the superconducting gap, such as specific heat or thermal conductivity, are dominated by the contribution from the substrate. Local probes like STM, being particularly sensitive to inhomogeneities in the sample, are often inconclusive about the symmetry of the gap function [12]. Measurements of the Meissner effect are also challenging in

*These authors contributed equally to this work.

Published by the American Physical Society under the terms of the Creative Commons Attribution 4.0 International license. Further distribution of this work must maintain attribution to the author(s) and the published article's title, journal citation, and DOI. atomically thin superconductors and require local magnetometry $[13,14]$. This calls for new experimental probes which can be used to diagnose the onset of superconductivity and elucidate the pairing symmetries in 2D materials.

In this Letter, we propose quantum noise spectroscopy by impurity spin qubits, such as nitrogen-vacancy (NV) centers in diamond, as a natural noninvasive wireless probe of $2 \mathrm{D}$ superconductivity. The probe qubit is initialized in a fully polarized state at a distance $z_{0}$ above our 2D sample [Fig. 1(a)]. Coupling to the noisy magnetic field created by thermal current fluctuations in the sample causes the qubit polarization to decay. The decay rate $\left(1 / T_{1}\right)$, studied as a function of experimentally tunable parameters such as qubit-probe distance $z_{0}$, probe frequency $\Omega$, and temperature $T$, furnishes valuable information about the nature of superconductivity in the $2 \mathrm{D}$ sample.

Specifically, we predict that the sharp reduction of noise due to suppression of transverse current fluctuations, stemming from the onset of a superconducting gap, allows one to detect the phase transition from a normal metal to a superconductor [Fig. 1(b)]. Second, we demonstrate that the nature of the superconducting gap (nodal vs non-nodal) can be deciphered by studying the noise as a function of temperature. Simultaneously, the scaling of the noise with probe-qubit distance can be used to study the nonlocal conductivity in the quasistatic limit $(\mathbf{q} \neq 0, \Omega \rightarrow 0)$, a regime complementary to existing probes (such as dc transport, $\mathrm{THz}$ spectroscopy, etc.). We elucidate these distinct scaling regimes in both clean and disordered superconductors, carefully accounting for additional modifications arising from superflow. Third, we illustrate that deep in the superconducting phase the noise 
(a)
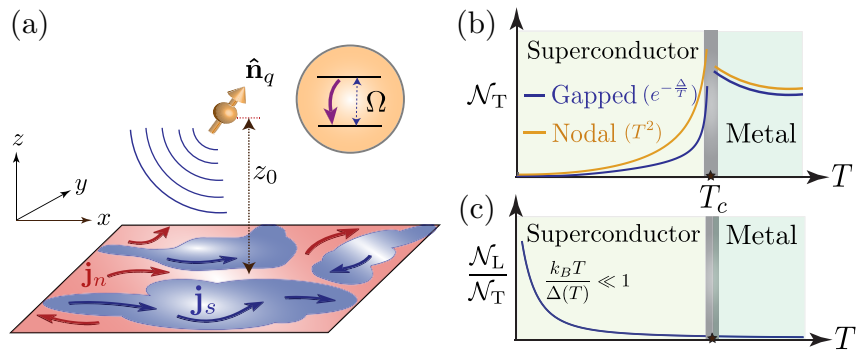

FIG. 1. (a) Schematic of the setup, with qubit at a distance $z_{0}$ from a $2 \mathrm{D}$ superconducting sample. The qubit polarization decays due to magnetic field noise created by fluctuating normal currents $\mathbf{j}_{n}$ and supercurrents $\mathbf{j}_{s}$ in the film. (b) Drop in the transverse noise $\mathcal{N}_{\mathrm{T}}$ when $T$ falls below $T_{c}$ for gapped and nodal superconductors (excluding effects of critical dynamics in narrow gray window around $T=T_{c}$ ). (c) Schematic of $\mathcal{N}_{\mathrm{L}} / \mathcal{N}_{\mathrm{T}}$ as a function of $T$, showing large enhancement at low $T$, making it possible to detect longitudinal collective modes.

is dominated by longitudinal current fluctuations [Fig. 1(c)], in sharp contrast to metals [15]. This owes its origin to the suppression of thermally excited quasiparticles at low temperatures, and allows us to detect collective longitudinal modes, such as gapless plasmons in monolayers and gapped Josephson plasmons in weakly interacting bilayer superconductors [16-20]. We conclude by providing realistic noise estimates for materials of recent interest, and argue that such measurements lie within experimental reach.

Setup and model. To detect magnetic noise, we consider an isolated impurity qubit placed at $\mathbf{r}_{0}=\left(0,0, z_{0}\right)$, i.e., at a distance $z_{0}$ above a homogeneous 2D sample in the $x-y$ plane [Fig. 1(a)]. The qubit Hamiltonian includes an intrinsic level splitting $\Omega$ along quantization axis $\hat{\mathbf{n}}_{q}$, and a linear coupling to the local magnetic field $\mathbf{B}\left(\mathbf{r}_{0}, t\right)$ (henceforth $\hbar=1$ ):

$$
H_{q}=\frac{\Omega}{2}\left(\hat{\mathbf{n}}_{q} \cdot \boldsymbol{\sigma}\right)+g \mu_{B} \mathbf{B}\left(\mathbf{r}_{0}, t\right) \cdot \boldsymbol{\sigma} .
$$

The depolarization rate of the qubit is directly proportional to the magnetic noise at the qubit location. This noise arises predominantly due to current fluctuations in the sample, assumed to be at thermal equilibrium at temperature $T$ [21]. By analyzing the relaxation rate of the qubit for different orientations of the quantization axis, one can isolate different components of the magnetic noise tensor $\mathcal{N}_{a b}(\Omega) \equiv$ $\frac{1}{2} \int_{-\infty}^{\infty} d t e^{i \Omega t}\left\langle\left\{B_{a}\left(\mathbf{r}_{0}, t\right), B_{b}\left(\mathbf{r}_{0}, 0\right)\right\}\right\rangle_{T}$ [15]. These in turn can be used to investigate two qualitatively different types of noise: (i) transverse noise $\mathcal{N}_{\mathrm{T}}$, arising from transverse current fluctuations $\left[\mathbf{j}_{\mathrm{T}}(\mathbf{q}) \perp \mathbf{q}\right]$ that do not create charge imbalance in the sample, is related to the reflection coefficient $r_{s}(\mathbf{q}, \Omega)$ for $s$-polarized electromagnetic (EM) waves; (ii) longitudinal noise $\mathcal{N}_{\mathrm{L}}$, arising from longitudinal current fluctuations $\left[\mathbf{j}_{\mathrm{L}}(\mathbf{q}) \| \mathbf{q}\right]$, is related to the reflection coefficient $r_{p}(\mathbf{q}, \Omega)$ for $p$-polarized EM waves ( $\mathbf{q}$ is the in-plane momentum). Reference [15] argued that the latter can be ignored in metals because $p$-polarized waves create charge density modulations that are disfavored by long-range Coulomb interactions. However, the presence of superconductivity can suppress $r_{s}$ at low temperatures and thereby provide a gateway to probe $r_{p}$. In what follows, we characterize magnetic noise due to

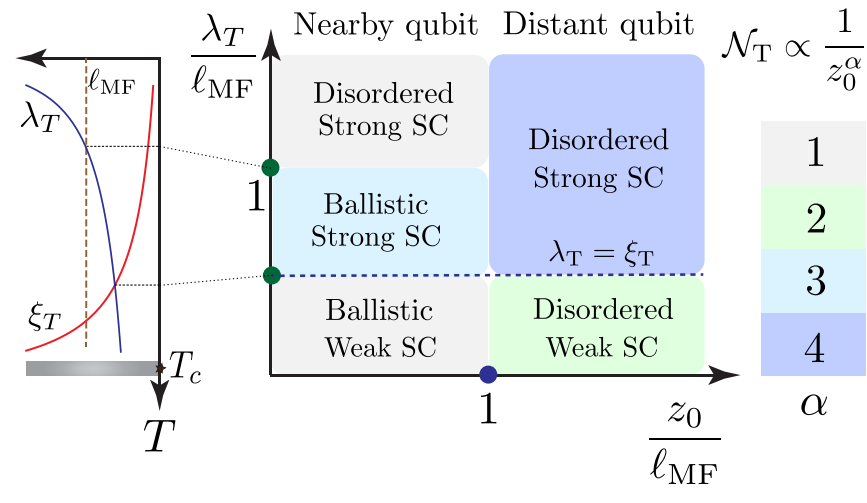

FIG. 2. Distance scaling of noise in different physical regimes in an $s$-wave superconductor, determined by the ratio of the thermal wavelength $\lambda_{T}$ or qubit-sample distance $z_{0}$ to the mean-free path $\ell_{\mathrm{MF}}$.

transverse and longitudinal current fluctuations in $2 \mathrm{D}$ superconductors and determine physical regimes where each contribution dominates. Here, we present our main physical results, but relegate computational details and additional discussion to a complementary paper [22].

Transverse noise. In the transverse sector, the noise is given in the experimentally relevant limit $\Omega \ll k_{B} T$ by $[15,22]$ (assuming in-plane rotational symmetry):

$$
\mathcal{N}_{\mathrm{T}}(\Omega) \approx \frac{\mu_{0} k_{B} T}{16 \pi z_{0}^{3} \Omega} \int_{0}^{\infty} d x x^{2} e^{-x} \operatorname{Im}\left\{r_{s}\left(\frac{x}{2 z_{0}}, \Omega\right)\right\},
$$

where $r_{s}(\mathbf{q}, \Omega)=-\left(1+\frac{2 i q}{\mu_{0} \Omega \sigma^{\mathrm{T}}(\mathbf{q}, \Omega)}\right)^{-1}$ is determined by the transverse conductivity $\sigma^{\mathrm{T}}(\mathbf{q}, \Omega)$. Therefore, the computation of noise reduces to evaluating $\sigma^{\mathrm{T}}(\mathbf{q}, \Omega)$ for the 2D sample of interest. For the sake of simplicity and physical transparency, we treat the superconductor within the two-fluid model [1]. The transverse conductivity consists of a dissipative part $\sigma_{n}^{\mathrm{T}}$ due to the normal fluid (quasiparticle excitations) and a reactive part due to transverse superflow, which can be computed using London's equation $\mathbf{j}_{\mathrm{T}, \mathrm{s}}=-\Lambda \mathbf{A}_{\mathrm{T}}=-\left(\frac{\Lambda}{i \Omega}\right) \mathbf{E}_{\mathrm{T}}$ :

$$
\sigma^{\mathrm{T}}(\mathbf{q}, \Omega)=\sigma_{n}^{\mathrm{T}}(\mathbf{q}, \Omega)-\frac{\Lambda}{i \Omega} .
$$

Within the phenomenological Ginzburg-Landau approach, $\Lambda \propto|\Delta(T)|^{2} \propto T_{c}-T, \Delta(T)$ being the superconducting gap. Therefore, as $T$ approaches $T_{c}$, the reactive part can be neglected, and the conductivity is dominated by the quasiparticle contribution:

$$
\mathcal{N}_{\mathrm{T}}(\Omega) \approx \frac{\mu_{0}^{2} k_{B} T}{16 \pi z_{0}^{2}} \operatorname{Re}\left\{\sigma_{n}^{\mathrm{T}}\left(\frac{1}{2 z_{0}}, \Omega\right)\right\} .
$$

In contrast, deep in the superconducting phase $\left(k_{B} T \ll \Delta(T)\right.$, indicated by "strong SC" in Fig. 2) at small frequency $\Omega$, the quasiparticle contribution is suppressed and conductivity is dominated by superflow ${ }^{1}$ :

$$
\mathcal{N}_{\mathrm{T}}(\Omega) \approx \frac{3 k_{B} T}{8 \pi z_{0}^{4} \Lambda^{2}} \operatorname{Re}\left\{\sigma_{n}^{\mathrm{T}}\left(\frac{3}{2 z_{0}}, \Omega\right)\right\} .
$$

\footnotetext{
${ }^{1}$ Strictly speaking, this formula is valid for $z_{0}$ being greater than the "Pearl length" $1 / \mu_{0} \Lambda$ [23]. See Ref. [22] for details.
} 
We see that in both limits, the essential signatures of the properties of the superconducting sample can be gleaned from $\operatorname{Re}\left\{\sigma_{n}^{\mathrm{T}}(q, \Omega)\right\}$, which we discuss next.

To calculate $\sigma_{n}^{\mathrm{T}}$ for the normal fluid, we resort to a microscopic description of the current-carrying quasiparticle excitations via the mean-field BCS Hamiltonian $H_{\mathrm{BCS}}$. For singlet superconductors, $H_{\mathrm{BCS}}$ is given in terms of the Nambu spinor $\Psi_{\mathbf{k}}=\left(c_{\mathbf{k}, \uparrow}, c_{-\mathbf{k}, \downarrow}^{\dagger}\right)^{T}$ (where $c_{\mathbf{k}, \sigma}$ are electron annihilation operators), electron dispersion $\xi_{\mathbf{k}}=\frac{k^{2}}{2 m}-\mu$, and gap function $\Delta_{\mathbf{k}}$ :

$$
H_{\mathrm{BCS}}=\sum_{\mathbf{k}} \Psi_{\mathbf{k}}^{\dagger} h_{\mathbf{k}} \Psi_{\mathbf{k}}, h_{\mathbf{k}}=\left(\begin{array}{cc}
\xi_{\mathbf{k}} & \Delta_{\mathbf{k}} \\
\Delta_{\mathbf{k}} & -\xi_{\mathbf{k}}
\end{array}\right) .
$$

Accordingly, the retarded Green's function is given by $G^{R}(\mathbf{k}, \omega)=\left(\omega+i 0^{+}+i \Gamma_{0}-h_{\mathbf{k}}\right)^{-1}$, where $\Gamma_{0}$ is the isotropic scattering rate of electrons at the Fermi surface due to uncorrelated quenched disorder, and it accounts for the broadening of the quasiparticle spectrum [24]. Denoting the transverse current density by $j_{\mathrm{T}}(\mathbf{q})=(\hat{z} \times \hat{\mathbf{q}}) \cdot \mathbf{j}(\mathbf{q})$, the conductivity can be evaluated within the standard linear response formalism [25], using the retarded transverse current-current correlation function $\Pi_{\mathrm{T}}^{R}(\mathbf{q}, t)=-i \theta(t)\left\langle\left[j_{\mathrm{T}}(\mathbf{q}, t), j_{\mathrm{T}}(-\mathbf{q}, 0)\right]\right\rangle / \mathcal{A}:$

$$
\begin{aligned}
& \operatorname{Re}\left[\sigma_{n}^{\mathrm{T}}(\mathbf{q}, \Omega)\right]=-\frac{\operatorname{Im}\left[\Pi_{\mathrm{T}}^{R}(\mathbf{q}, \Omega)\right]}{\Omega} \\
& \stackrel{\beta \Omega \ll 1}{\longrightarrow} \frac{e^{2}}{2 \pi} \int_{\mathbf{k}, \omega} v_{\mathrm{T}}^{2} \operatorname{Tr}\left[A\left(\mathbf{k}_{-}, \omega\right) A\left(\mathbf{k}_{+}, \omega_{+}\right)\right]\left(-\frac{\partial n_{F}}{\partial \omega}\right),
\end{aligned}
$$

where $\boldsymbol{v}_{\mathrm{T}}(\hat{k})=v_{F}(\hat{q} \times \hat{k})$ is the transverse component of Fermi velocity, $\mathbf{k}_{ \pm}=\mathbf{k} \pm \mathbf{q} / 2, \omega_{+}=\omega+\Omega, A(\mathbf{k}, \Omega)=$ $-\operatorname{Im}\left[G^{R}(\mathbf{k}, \Omega)\right] / \pi$ is the spectral function, $\mathcal{A}$ is the area of the sample, and $n_{F}(\omega)=[\exp (\beta \omega)+1]^{-1}$ is the Fermi function $\left(\beta=1 / k_{B} T\right)$. A key point to note is that as a consequence of momentum and energy selectivity, the qubit probe is most sensitive to $\sigma_{n}^{\mathrm{T}}\left(q \sim 1 / z_{0}, \Omega \approx 0\right)$ since for realistic experimental scenarios the relevant dimensionless variable $\Omega /\left(v_{F} q\right) \sim \Omega z_{0} / v_{F} \ll 1$. This is a completely distinct order of limits from the usual computation of dc conductivity, where one first takes $\mathbf{q} \rightarrow 0$ and then $\Omega \rightarrow 0$. As a consequence, our qubit probe opens the door to studying the finite-momentum low-frequency transverse conductivity, which yields information about intrinsic behavior that is typically inaccessible otherwise.

The behavior of $\sigma_{n}^{\mathrm{T}}(\mathbf{q}, \Omega)$ is generally quite complex, and is determined by the rich interplay of several different length scales: (i) the quasiparticle mean-free path $\ell_{\mathrm{MF}}=v_{F} /\left(2 \Gamma_{0}\right)$, (ii) the qubit-sample distance $z_{0}$, (iii) the quasiparticle thermal wavelength $\lambda_{T}=v_{F} / k_{B} T$, and (iv) the superconducting coherence length $\xi_{T} \equiv v_{F} / \Delta(T)$. Varying these parameters by tuning $z_{0}$ and $T$ results in various crossovers in the behavior of $\sigma_{n}^{\mathrm{T}}\left(q \sim 1 / z_{0}, 0\right)$, which can then be used to infer important properties of the superconducting (SC) state. Before getting into the details of these crossovers, we highlight the essential gross features: Above $T_{c}, \sigma_{n}^{\mathrm{T}}(\mathbf{q}, 0)$ is approximately independent of $T$, resulting in $\mathcal{N}_{\mathrm{T}}^{h} \propto T$. Upon cooling through $T_{c}$, a significant suppression of noise occurs, providing a clear indication of the superconducting transition. In an $s$-wave su- perconductor, quasiparticle excitations are suppressed by the gap, so that the noise decreases exponentially as $e^{-\Delta(T) / k_{B} T}$. In a $d$-wave supercondcutor, the presence of nodal quasiparticles yields a $T^{2}$ behavior. Distinct temperature scalings of the noise therefore provide a way to distinguish superconducting order parameters.

In addition to temperature dependence, the scaling of noise with distance is different for nodal vs non-nodal superconductors: In the temperature regime $k_{B} T \lesssim \Gamma_{0}$, we find $\mathcal{N}_{\mathrm{T}} \propto 1 / z_{0}$ in the $s$-wave case, while $\mathcal{N}_{\mathrm{T}} \propto 1 / z_{0}^{2}$ for the $d$-wave one. We will now elaborate further on the behavior $\sigma_{n}^{\mathrm{T}}\left(q \sim 1 / z_{0}, 0\right)$, appealing to physical arguments to demonstrate how the distance scaling of noise can be used to distinguish different transport regimes. All our conclusions are verified by explicit analytic and numerical evaluation of noise across arbitrary parameter regimes [22].

We first consider 2D $s$-wave superconductors in the clean limit, where the thermal wavelength $\lambda_{T}$ is much smaller than the mean-free path $\ell_{\mathrm{MF}}$, or equivalently $\Gamma_{0} \lesssim k_{B} T$. In this limit, the spectral function $A(\mathbf{k}, \omega)$ is not sufficiently smoothed out by disorder on the scale of $|\omega| \lesssim k_{B} T$, the frequency range where $n_{F}^{\prime}(\omega)$ is significant. Hence, it is useful to think of the conductivity as arising from sharp transitions of quasiparticles near the Fermi surface across an energy shell of width $\Omega$ and a momentum transfer $\mathbf{q}$. The phase space of such excitations is constrained by the geometry of the Fermi surface and grows as $1 / q$. Accordingly, at low temperatures $k_{B} T \ll \Delta(T)$, the conductivity scales as $\sigma_{n}^{\mathrm{T}}(\mathbf{q}, 0) \propto$ $e^{-\beta \Delta}\left(q T \xi_{T}\right)^{-1}$. Considering additional suppression due to the superflow in this regime, Eq. (5) predicts $\mathcal{N}_{\mathrm{T}} \propto 1 / z_{0}^{3}$. On increasing $T$, as the Cooper pairs get loosely bound and $\xi_{T}$ crosses the thermal length $\lambda_{T}$, we recover the conductivity of a ballistic Fermi liquid by simply replacing $\xi_{T}$ by $\lambda_{T}$ in the above expression, i.e., $\sigma_{n}^{\mathrm{T}}(\mathbf{q}, 0) \propto\left(q T \lambda_{T}\right)^{-1}=\left(q v_{F}\right)^{-1}$ and $\mathcal{N}_{\mathrm{T}} \propto 1 / z_{0}[26]$.

In the dirty limit $\Gamma_{0} \gtrsim k_{B} T$, the spectral function is smooth on the scale of $\omega \lesssim k_{B} T$, and we can replace the Fermifunction derivative by a delta function at $\omega=0$. In this disordered superconductor, the conductivity is determined by the product of spectral functions near the Fermi surface [see Eq. (7)]. If we lower the sample-probe distance to probe large momenta, i.e., $q \ell_{\mathrm{MF}}$ and $q \xi_{T}$ are both large (but $q \ll k_{F}$ ), then the product $A\left(\mathbf{k}_{+}, 0\right) A\left(\mathbf{k}_{-}, 0\right) \sim 1 /\left(v_{F} q\right)^{4}$ for $|\mathbf{k}| \approx k_{F}$. The dominant contribution to transverse conductivity comes from an annular region of width $q$ and circumference $2 \pi k_{F}$ around the circular Fermi surface. Accordingly, the conductivity scales as $2 \pi k_{F} \times q \times 1 / q^{4} \sim k_{F} / q^{3}$, and Eq. (5) predicts the noise decreases as $1 / z_{0}$. On approaching the opposite limit $z_{0} \gg \ell_{\mathrm{MF}}$, the probe is sensitive to multiple scattering events, resulting in the usual metallic behavior, where $\sigma_{n}^{\mathrm{T}}(\mathbf{q}, 0)$ is independent of momentum and $\mathcal{N}_{\mathrm{T}} \propto 1 / z_{0}^{4}$. The noise scalings in different regimes are summarized in Fig. 2, along with the behavior in the regime $\Delta(T) \lesssim k_{B} T$, which we refer to as the "weak superconductor (weak SC)." In the latter regime, the scaling is the same as that in the metallic phase above $T_{c}$.

Next, we consider $d$-wave superconductors with the gap function $\Delta_{\mathbf{k}}(T)=\Delta_{0}(T)\left(k_{x}^{2}-k_{y}^{2}\right)$. We find that the distinct temperature scaling of the noise carries signatures of gapless Dirac quasiparticles at the nodes $k=k_{F}, k_{x}= \pm k_{y}$. In the clean (ballistic) limit, at low $k_{B} T \ll \Delta_{0}(T)$, the noise scales 
as $T^{2}$ due to the power-law low-energy density of states at the Dirac cones. As $T \rightarrow T_{c}^{-}$, the noise increases steeply as $v_{\Delta}^{-1} \ln \left(v_{F} / v_{\Delta}\right)$, where the gap velocity $v_{\Delta}=\left|\partial_{\mathbf{k}} \Delta_{\mathbf{k}}\right|_{\text {node }}$ decreases as $\left|T_{c}-T\right|^{1 / 2}$ within mean-field theory. This logarithmic correction owes its origin to the anisotropy between the Fermi velocity $v_{F}$ and the gap velocity $v_{\Delta}$ : The dominant contribution comes from the tips of anisotropic Dirac cones with large density of states. In the dirty limit, the disorderinduced finite density of states at zero energy leads to a linear in $T$ scaling of the noise, as the conductivity $\sigma_{n}^{\mathrm{T}}(\mathbf{q}, \Omega=0)$ approaches a constant nonsingular value of $e^{2} v_{F} / \pi^{2} v_{\Delta}$ at small $q$ [24].

The physics of distance scalings remains roughly the same as for the $s$-wave case, with the exception of dirty superconductors at sample-probe distances smaller than the mean-free path. The available phase space for excitations constitutes a patch of area $q^{2}$ around each nodal point, implying $\sigma_{n}^{\mathrm{T}}(\mathbf{q}, 0) \propto$ $q^{2} \times 1 / q^{4}=1 / q^{2}$. In this limit, the noise scales as $1 / z_{0}^{2}$, up to logarithmic corrections, and can be used to differentiate nodal and non-nodal superconducting gap functions.

Noise from longitudinal collective modes. As remarked, at low temperatures, the quasiparticle noise in the transverse sector is heavily suppressed, and longitudinal fluctuations can dominate. Longitudinal noise is given by [22]

$$
\mathcal{N}_{\mathrm{L}}(\Omega)=\frac{k_{B} T \epsilon \mu_{0} \Omega}{16 \pi c^{2} z_{0}} \int_{0}^{\infty} d x e^{-x} \operatorname{Im}\left\{r_{p}\left(\frac{x}{2 z_{0}}, \Omega\right)\right\},
$$

where $r_{p}(\mathbf{q}, \Omega)=\left(1+\frac{2 \epsilon \epsilon_{0} \Omega}{i q \sigma^{\circ}(\mathbf{q}, \Omega)}\right)^{-1}$ and $\epsilon$ is the highfrequency dielectric constant of the encapsulating medium. The longitudinal conductivity $\sigma^{\mathrm{L}}(\mathbf{q}, \Omega)$ can be evaluated by considering longitudinal current fluctuations via Maxwell's equations and time-dependent Ginzburg-Landau theory for the superconducting order parameter [1]. For a 2D sample, such an analysis yields a collective plasmon mode with a gapless dispersion: $\omega_{\|}(q) \approx \sqrt{q \Lambda /\left(2 \epsilon \epsilon_{0}\right)}$ [22]. This collective excitation represents an evanescent wave that couples three-dimensional fluctuations of the electromagnetic field to two-dimensional quasiparticle currents and order-parameter fluctuations. Interestingly, we find that the reflection coefficient $r_{p}$, evaluated for evanescent waves, exhibits a sharp peak at the resonance frequency $\Omega=\omega_{\|}(q)$. Therefore, the longitudinal noise can become resonantly enhanced when the probe distance $z_{0}$ is decreased to cross the longitudinal spectrum (one could also vary the probe frequency $\Omega$ at fixed $z_{0}$ ):

$$
\mathcal{N}_{\mathrm{L}}(\Omega) \approx \mathcal{N}^{*}(\Omega) \exp \left(-\frac{4 \epsilon \epsilon_{0} z_{0} \Omega^{2}}{\Lambda}\right),
$$

where $\mathcal{N}^{*}(\Omega)=k_{B} T \epsilon^{2} \Omega^{3} /\left(4 \Lambda c^{4}\right)$. We conclude that although $\sigma_{n}^{\mathrm{L}}$ can be vanishingly small at low temperatures due to superconductivity, the noise still gets a substantial contribution from the longitudinal collective modes. In particular, $\mathcal{N}_{\mathrm{L}}(\Omega) / \mathcal{N}^{*}(\Omega)$ is suppressed for large sample-probe distances, whereas it saturates to a finite value for $z_{0} \lesssim z^{*}(\Omega)$, defined by $\Omega=\omega_{\|}\left(1 / 2 z^{*}\right)$, i.e., upon crossing the plasmon branch, as illustrated in Fig. 3. We also note that, in bilayers, interlayer Josephson plasmons lead to an additional resonance in the longitudinal noise (see Ref. [22] for further discussion).

Experimental feasibility. To be concrete, let us consider $\mathrm{NV}$ centers in diamond [27-31]. The intrinsic level splitting is

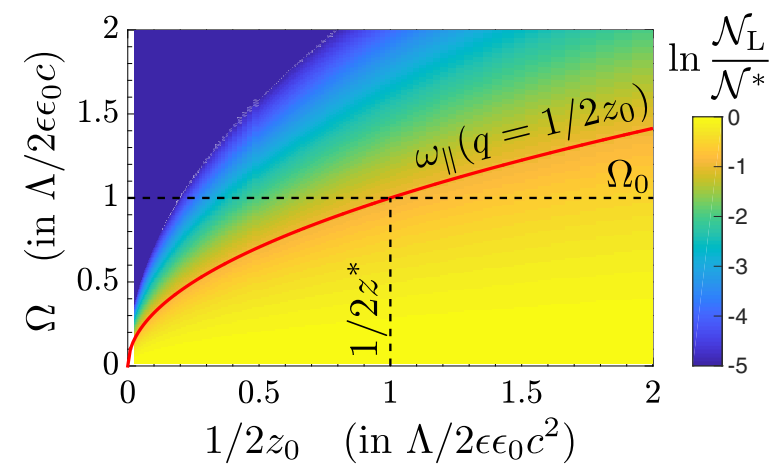

FIG. 3. Plasmon contribution to the low-temperature noise. Shown is $\ln \left[\mathcal{N}_{\mathrm{L}}\left(\Omega, z_{0}\right) / \mathcal{N}^{*}(\Omega)\right]$ as a function of $\Omega$ and $z_{0}$. For large $z_{0} \gg z^{*}(\Omega) \equiv 4 \epsilon \epsilon_{0} \Lambda / \Omega^{2}$ [such that $\Omega=\omega_{\|}\left(1 / 2 z^{*}\right)$ ], the noise is suppressed and it quickly saturates upon crossing the plasmon dispersion (solid red line), i.e., for $z_{0} \lesssim z^{*}(\Omega)$.

$\Omega / 2 \pi=2.87 \mathrm{GHz}(\approx 0.1 \mathrm{~K})$, the smallest energy scale in the problem. This justifies taking $\Omega \rightarrow 0$ in our calculations. For magic-angle moiré graphene, $T_{c} \approx 3-4 \mathrm{~K}[2,5,6]$. Assuming sufficient disorder (due to local strain [32]), we approximate $\sigma^{\mathrm{T}}(q) \approx \sigma(q=0) \approx 10^{-2} S$ in the metallic state just above $T_{c}$. For $z_{0}=5 \mathrm{~nm}$, we can therefore estimate $\mathcal{N}_{\mathrm{T}} \approx \frac{k_{B} T \mu_{0}^{2} \sigma_{n}^{\mathrm{T}}}{16 \pi z_{0}^{2}} \approx$ $120 \mathrm{pT}^{2} / \mathrm{Hz}$. Accordingly, the depolarization rate of the qubit is given by [15] $1 / T_{1}=\sqrt{S(S+1)} g^{2} \mu_{B}^{2} \mathcal{N}_{\mathrm{T}} /\left(2 \hbar^{2}\right) \approx 3.2 \mathrm{~s}^{-1}$ ( $S=1$ for NV). The lifetime in the presence of a sample is therefore much shorter than the intrinsic lifetime of an isolated $\mathrm{NV}$ center, which can exceed $10^{2} \mathrm{~s}$ below $10 \mathrm{~K}[33,34]$. Further, within mean-field theory $\Delta(T) \approx 3 k_{B} T_{c} \sqrt{1-T / T_{c}}$ [1], implying that on decreasing $T$ from $T_{c}$ to $T_{c} / 2,1 / T_{1}$ drops roughly by a factor of $e^{\beta \Delta(T)} \approx 70$, making the change easily detectable. Comparable parameters apply to TMDs, which exhibit a variety of interesting superconducting behaviors [35], such as "Ising superconductivity." The foregoing estimates show that the detection of the ordering transition is realistic within the existing experimental tools. However, to detect the longitudinal collective modes, one might need to use high- $\epsilon$ materials, such as $\mathrm{SrTiO}_{3}$ with $\epsilon \approx 10000$ [36]. Another way to enhance this signal is to increase the probe frequency $\Omega$ via the application of an in-plane magnetic field [22].

Discussion. Our work establishes a complimentary route to detect and study 2D superconductivity via noise magnetometry and offers a window into the elusive Coulomb-interactiondominated longitudinal collective modes in superconductors. The ability to probe across a wide range of length scales allows our technique to investigate inhomogeneous materials and to potentially understand how superconductivity differs across samples. Our calculations are valid away from the critical regime, where critical current fluctuations might lead to an appreciable enhancement of noise, facilitating easier experimental detection. This exciting direction deserves further investigation, though we note that this critical temperature window is typically relatively narrow in superconductors.

While our qubit probe is also sensitive to noise arising from spin fluctuations, spin noise in singlet superconductors is suppressed relative to charge noise by an additional factor of $1 /\left(k_{F} z_{0}\right)^{2}[15]$ in the nearly metallic regime and 
by $\left(\mu_{0} \mu_{B} \Lambda / e v_{F}\right)^{2}$ deep in the superconducting phase [22]. Further, in contrast to NMR, there is no anomalous enhancement of spin noise (relative to current noise), as the nonlocal response leads to smearing out the singular density of states that is responsible for the Hebel-Slichter peak [22]. However, in superconductors with spontaneously broken spin-rotation symmetry (in materials with negligible spin-orbit coupling), spin noise may receive resonant enhancement from spin waves [37,38].

Previous works have developed theoretical underpinnings of probing different regimes of electron transport in metallic systems, as well as magnetic phases and their phase transitions in insulators, using quantum impurity probes [15,37,39]. On the experimental front, noise measurements using single-spin qubits have recently been used to detect a plethora of novel quantum phenomena, including nonlocal conductivity in metallic silver [40], magnetic transitions in metallic Gd [41], chiral magnons [42], spin diffusion in antiferromagnets [43], and electron-phonon instabilities in graphene [44]. With the discovery of superconductivity in several new 2D materials, the qubit probe appears ideally suited to unravel their properties. The noninvasive nature of the qubit, with fully optical ini- tialization and readout capabilities, adds to its appeal. Finally, we note that current fluctuations in the sample also provide electric field noise at the qubit location $[45,46]$ and alternative qubits $[47,48]$ are more susceptible to this kind of noise. Their response can be studied by a generalization of the theoretical framework developed here and is left for future work.

Acknowledgments. We thank T. Andersen, B. Dwyer, S. Hsieh, S. Kolkowitz, V. Manucharian, J. F. Rodriguez-Nieva, E. Urbach, R. Xue, A. Yacoby, and C. Zu for helpful conversations. S.C. was supported by the ARO through the Anyon Bridge MURI program (Grant No. W911NF-17-1-0323) via M. P. Zaletel, and the U.S. DOE, Office of Science, Office of Advanced Scientific Computing Research, under the Accelerated Research in Quantum Computing (ARQC) program via N.Y. Yao. P.E.D, I.E., and E.D. were supported by Harvard-MIT CUA, AFOSR-MURI: Photonic Quantum Matter Award No. FA95501610323, Harvard Quantum Initiative, and AFOSR Grant No. FA9550-21-1-0216. N.Y.Y. acknowledges support from U.S. DOE, Office of Science, Office of Advanced Scientific Computing Research Quantum Testbed Program. Support from the Gordon and Betty Moore Foundation is also gratefully acknowledged.
[1] Michael Tinkham, Introduction to Superconductivity (Dover, New York, 2004).

[2] Y. Cao, V. Fatemi, S. Fang, K. Watanabe, T. Taniguchi, E. Kaxiras, and P. Jarillo-Herrero, Unconventional superconductivity in magic-angle graphene superlattices, Nature (London) 556, 43 (2018).

[3] X. Lu, P. Stepanov, W. Yang, M. Xie, Mohammed Ali Aamir, I. Das, C. Urgell, K. Watanabe, T. Taniguchi, G. Zhang et al., Superconductors, orbital magnets and correlated states in magic-angle bilayer graphene, Nature (London) 574, 653 (2019).

[4] M. Yankowitz, S. Chen, H. Polshyn, Y. Zhang, K. Watanabe, T. Taniguchi, D. Graf, A. F. Young, and C. R. Dean, Tuning superconductivity in twisted bilayer graphene, Science $\mathbf{3 6 3}$, 1059 (2019).

[5] J. M. Park, Y. Cao, K. Watanabe, T. Taniguchi, and P. Jarillo-Herrero, Tunable strongly coupled superconductivity in magic-angle twisted trilayer graphene, Nature (London) 590, 249 (2021)

[6] Z. Hao, A. M. Zimmerman, P. Ledwith, E. Khalaf, D. H. Najafabadi, K. Watanabe, T. Taniguchi, A. Vishwanath, and P. Kim, Electric field tunable superconductivity in alternating twist magic-angle trilayer graphene, Science 371, 1133 (2021).

[7] W. Shi, J. Ye, Y. Zhang, R. Suzuki, M. Yoshida, J. Miyazaki, N. Inoue, Y. Saito, and Y. Iwasa, Superconductivity series in transition metal dichalcogenides by ionic gating, Sci. Rep. 5, 12534 (2015).

[8] J.-F. Ge, Z.-L. Liu, C. Liu, C.-L. Gao, D. Qian, Q.-K. Xue, Y. Liu, and J.-F. Jia, Superconductivity above $100 \mathrm{k}$ in single-layer fese films on doped $\mathrm{SrTiO}_{3}$, Nat. Mater. 14, 285 (2015).

[9] Q.-Y. Wang, Z. Li, W.-H. Zhang, Z.-C. Zhang, J.-S. Zhang, W. Li, H. Ding, Y.-B. Ou, P. Deng, K. Chang, J. Wen, C.-L. Song, K. He, J.-F. Jia, S.-H. Ji, Y.-Y. Wang, L.-L. Wang, X. Chen, X.C. Ma, and Qi-Kun Xue, Interface-Induced High-Temperature
Superconductivity in Single Unit-Cell FeSe Films on $\mathrm{SrTiO}_{3}$, Chin. Phys. Lett. 29, 037402 (2012).

[10] C.-L. Song, Y.-L. Wang, Y.-P. Jiang, Z. Li, L. Wang, K. He, X. Chen, X.-C. Ma, and Q.-K. Xue, Molecular-beam epitaxy and robust superconductivity of stoichiometric fese crystalline films on bilayer graphene, Phys. Rev. B 84, 020503(R) (2011).

[11] A. Allain, J. Kang, K. Banerjee, and A. Kis, Electrical contacts to two-dimensional semiconductors, Nat. Mater. 14, 1195 (2015).

[12] Q. Gu, Y. Li, S. Wan, H. Li, W. Guo, H. Yang, Q. Li, X. Zhu, X. Pan, Y. Nie et al., Single particle tunneling spectrum of superconducting $\mathrm{Nd}_{1-x} \mathrm{Sr}_{x} \mathrm{NiO}_{2}$ thin films, Nat. Commun. 11, 6027 (2020).

[13] Z. Zhang, Y.-H. Wang, Q. Song, C. Liu, R. Peng, K. A. Moler, D. Feng, and Y. Wang, Onset of the meissner effect at $65 \mathrm{~K}$ in FeSe thin film grown on Nb-doped $\mathrm{SrTiO}_{3}$ substrate, Sci. Bull. 60, 1301 (2015).

[14] Y. Xu, Y. Yu, Y. Y. Hui, Y. Su, J. Cheng, H.-C. Chang, Y. Zhang, Y. R. Shen, and C. Tian, Mapping dynamical magnetic responses of ultrathin micron-size superconducting films using nitrogen-vacancy centers in diamond, Nano Lett. 19, 5697 (2019).

[15] K. Agarwal, R. Schmidt, B. Halperin, V. Oganesyan, G. Zaránd, M. D. Lukin, and E. Demler, Magnetic noise spectroscopy as a probe of local electronic correlations in two-dimensional systems, Phys. Rev. B 95, 155107 (2017).

[16] P. W. Anderson, Random-phase approximation in the theory of superconductivity, Phys. Rev. 112, 1900 (1958).

[17] I. O. Kulik and I. K. Yanson, The josephson effect in superconductive tunneling structures, Am. J. Phys. 42, 799 (1974).

[18] A. Barone and G. Paterno, Physics and Applications of the Josephson Effect (Wiley, New York, 1982).

[19] L. N. Bulaevskii, M. Zamora, D. Baeriswyl, H. Beck, and J. R. Clem, Time-dependent equations for phase differences and a 
collective mode in Josephson-coupled layered superconductors, Phys. Rev. B 50, 12831 (1994).

[20] Z. Sun, M. M. Fogler, D. N. Basov, and A. J. Millis, Collective modes and terahertz near-field response of superconductors, Phys. Rev. Res. 2, 023413 (2020).

[21] L. S. Langsjoen, A. Poudel, M. G. Vavilov, and R. Joynt, Qubit relaxation from evanescent-wave Johnson noise, Phys. Rev. A 86, 010301(R) (2012).

[22] P. E. Dolgirev, S. Chatterjee, I. Esterlis, A. A. Zibrov, M. D. Lukin, N. Y. Yao, and E. Demler, Characterizing two-dimensional superconductivity via nanoscale noise magnetometry with single-spin qubits, Phys. Rev. B 105, 024507 (2022).

[23] J. Pearl, Current distribution in superconducting films carrying quantized fluxoids, Appl. Phys. Lett. 5, 65 (1964).

[24] A. C. Durst and P. A. Lee, Impurity-induced quasiparticle transport and universal-limit wiedemann-franz violation in $d$-wave superconductors, Phys. Rev. B 62, 1270 (2000).

[25] A. Altland and B. D. Simons, Condensed Matter Field Theory (Cambridge University Press, Cambridge, 2010).

[26] J. Y. Khoo, F. Pientka, and I. Sodemann, The universal shear conductivity of Fermi liquids and spinon Fermi surface states and its detection via spin qubit noise magnetometry, New J. Phys. 23, 113009 (2021).

[27] S. Hong, M. S Grinolds, L. M. Pham, D. Le Sage, L. Luan, R. L. Walsworth, and A. Yacoby, Nanoscale magnetometry with nv centers in diamond, MRS Bull. 38, 155 (2013).

[28] M. S. Grinolds, S. Hong, P. Maletinsky, L. Luan, M. D. Lukin, R. L. Walsworth, and A. Yacoby, Nanoscale magnetic imaging of a single electron spin under ambient conditions, Nat. Phys. 9, 215 (2013).

[29] L. Rondin, J.-P. Tetienne, T. Hingant, J.-F. Roch, P. Maletinsky, and V. Jacques, Magnetometry with nitrogenvacancy defects in diamond, Rep. Prog. Phys. 77, 056503 (2014).

[30] F. Casola, T. van der Sar, and A. Yacoby, Probing condensed matter physics with magnetometry based on nitrogenvacancy centres in diamond, Nat. Rev. Mater. 3, 17088 (2018).

[31] P. Stano, J. Klinovaja, A. Yacoby, and D. Loss, Local spin susceptibilities of low-dimensional electron systems, Phys. Rev. B 88, 045441 (2013).

[32] N. P. Kazmierczak, M. Van Winkle, C. Ophus, K. C. Bustillo, S. Carr, H. G. Brown, J. Ciston, T. Taniguchi, K. Watanabe, and D. K. Bediako, Strain fields in twisted bilayer graphene, Nat. Mater. 20, 956 (2021).

[33] A. Jarmola, V. M. Acosta, K. Jensen, S. Chemerisov, and D. Budker, Temperature- and magnetic-field-dependent longitudinal spin relaxation in nitrogen-vacancy ensembles in diamond, Phys. Rev. Lett. 108, 197601 (2012).

[34] T. de Guillebon, B. Vindolet, J.-F. Roch, V. Jacques, and L. Rondin, Temperature dependence of the longitudinal spin relaxation time $T_{1}$ of single nitrogen-vacancy centers in nanodiamonds, Phys. Rev. B 102, 165427 (2020).
[35] S. Manzeli, D. Ovchinnikov, D. Pasquier, O. V. Yazyev, and A. Kis, 2D transition metal dichalcogenides, Nat. Rev. Mater. 2, 17033 (2017).

[36] L. Veyrat, C. Déprez, A. Coissard, X. Li, F. Gay, K. Watanabe, T. Taniguchi, Z. Han, B. A. Piot, H. Sellier, and B. Sacépé, Helical quantum Hall phase in graphene on $\mathrm{SrTiO}_{3}$, Science 367, 781 (2020).

[37] S. Chatterjee, J. F. Rodriguez-Nieva, and E. Demler, Diagnosing phases of magnetic insulators via noise magnetometry with spin qubits, Phys. Rev. B 99, 104425 (2019).

[38] J. F. Rodriguez-Nieva, D. Podolsky, and E. Demler, Hydrodynamic sound modes and Galilean symmetry breaking in a magnon fluid, arXiv:1810.12333.

[39] J. F. Rodriguez-Nieva, K. Agarwal, T. Giamarchi, B. I. Halperin, M. D. Lukin, and E. Demler, Probing onedimensional systems via noise magnetometry with single spin qubits, Phys. Rev. B 98, 195433 (2018).

[40] S. Kolkowitz, A. Safira, A. A. High, R. C. Devlin, S. Choi, Q. P. Unterreithmeier, D. Patterson, A. S. Zibrov, V. E. Manucharyan, H. Park et al., Probing johnson noise and ballistic transport in normal metals with a single-spin qubit, Science 347, 1129 (2015).

[41] S. Hsieh, P. Bhattacharyya, C. Zu, T. Mittiga, T. J. Smart, F. Machado, B. Kobrin, T. O. Höhn, N. Z. Rui, M. Kamrani, S. Chatterjee, S. Choi, M. Zaletel, V. V. Struzhkin, J. E. Moore, V. I. Levitas, R. Jeanloz, and N. Y. Yao, Imaging stress and magnetism at high pressures using a nanoscale quantum sensor, Science 366, 1349 (2019).

[42] A. Rustagi, I. Bertelli, T. van der Sar, and P. Upadhyaya, Sensing chiral magnetic noise via quantum impurity relaxometry, Phys. Rev. B 102, 220403(R) (2020).

[43] H. Wang, S. Zhang, N. J. McLaughlin, B. Flebus, M. Huang, Y. Xiao, E. E. Fullerton, Y. Tserkovnyak, and C. R. Du, Quantum Sensing of Spin Transport Properties of an Antiferromagnetic Insulator, arXiv:2011.03905.

[44] T. I. Andersen, B. L. Dwyer, J. D. Sanchez-Yamagishi, J. F. Rodriguez-Nieva, K. Agarwal, K. Watanabe, T. Taniguchi, E. A. Demler, P. Kim, H. Park et al., Electron-phonon instability in graphene revealed by global and local noise probes, Science 364, 154 (2019).

[45] B. A. Myers, A. Ariyaratne, and A. C. Bleszynski Jayich, Double-quantum spin-relaxation limits to coherence of nearsurface nitrogen-vacancy centers, Phys. Rev. Lett. 118, 197201 (2017).

[46] R. Sahay, S. Hsieh, E. Parsonnet, L. W. Martin, R. Ramesh, N. Y. Yao, and S. Chatterjee, Noise Electrometry of Polar and Dielectric Materials, arXiv:2111.09315.

[47] A. Gottscholl, M. Kianinia, V. Soltamov, S. Orlinskii, G. Mamin, C. Bradac, C. Kasper, K. Krambrock, A. Sperlich, M. Toth et al., Initialization and read-out of intrinsic spin defects in a van der Waals crystal at room temperature, Nat. Mater. 19, 540 (2020).

[48] J. P. Tetienne, Quantum sensors go flat, Nat. Phys. 17, 1074 (2021). 